\title{
Long-lived coherences for line-narrowing in high-field NMR
}

\section{Riddhiman Sarkar ${ }^{\mathrm{a}, \mathrm{b}}$, Puneet Ahuja ${ }^{\mathrm{b}, *}$, Paul R. Vasos ${ }^{\mathrm{b}, \mathrm{c}}$, Aurélien Bornet ${ }^{\mathrm{b}}$, Olivier Wagnières ${ }^{\mathrm{b}}$, Geoffrey Bodenhausen b,d,e,f}

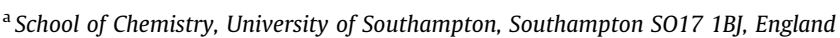

${ }^{\mathrm{b}}$ Institut des Sciences et Ingénierie Chimiques, Ecole Polytechnique Fédérale de Lausanne, EPFL, Batochime, 1015 Lausanne, Switzerland

' Université Paris Descartes, UMR 8601 CNRS, 45, rue des Saints Pères 75006, Paris, France

${ }^{\mathrm{d}}$ Université Pierre-et-Marie Curie, Paris, France

e Département de Chimie, Ecole Normale Supérieure, 24 Rue Lhomond, 75231, Paris Cedex 05, France

${ }^{\mathrm{f}}$ CNRS, UMR 7203, France

\section{A R T I C L E I N F O}

\section{Article history:}

Received 8 June 2010

Accepted 14 October 2010

Available online 21 October 2010

\section{Keywords:}

Long-lived states

Long-lived coherences

Line narrowing

Coherent superpositions

Transverse relaxation

\section{Contents}

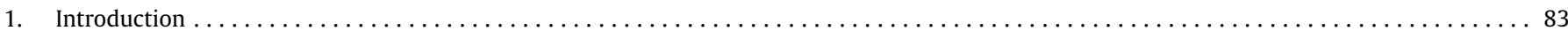

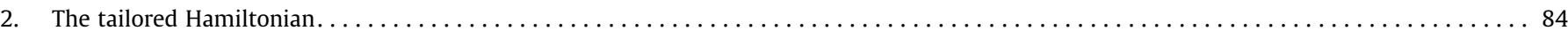

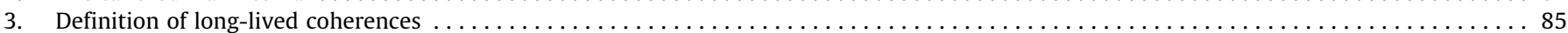

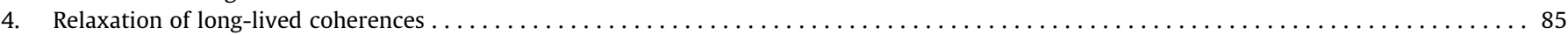

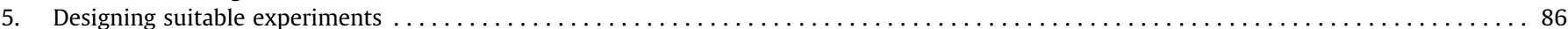

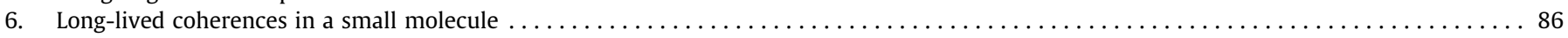

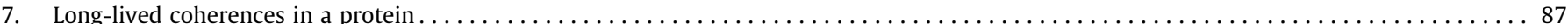

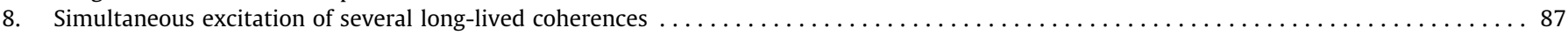

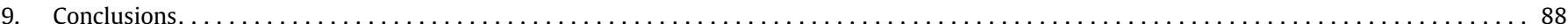

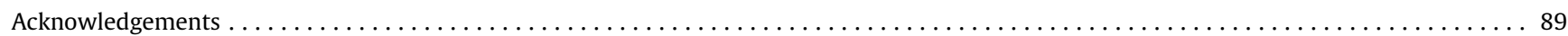

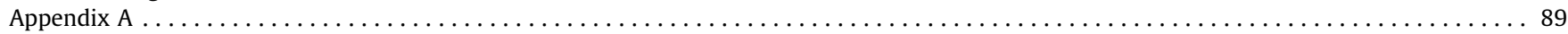

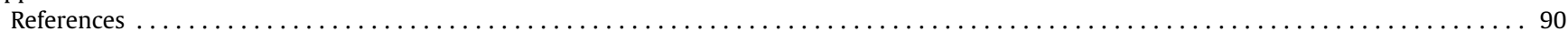

\section{Introduction}

In conventional nuclear magnetic resonance spectroscopy (NMR), there is a well-known cascade of causes that lead to linebroadening effects: the larger the size of the molecules under investigation, the slower the rotational diffusion, the stronger the low-frequency components of the spectral density functions, the

\footnotetext{
* Corresponding author.

E-mail address: puneet.ahuja@epfl.ch (P. Ahuja).
}

faster the transverse relaxation, hence the broader the spectral lines. This not only holds for 'conventional' spectroscopy of allowed transitions, i.e., for the observation of single-quantum transitions, but also for zero- and multiple-quantum spectra. The line broadening associated with slow tumbling makes NMR of large molecules and supramolecular assemblies difficult.

If we consider a pair of coupled spin- $1 / 2$ nuclei I and $S$ that are reasonably isolated from unpaired electrons and other nuclei with significant magnetic moments, the longitudinal ('spin-lattice') relaxation rate $R_{1}=1 / T_{1}$ and the transverse ('spin-spin') relaxation 
rate $R_{2}=1 / T_{2}$ of single-quantum coherences (SQCs) are predominantly due to the dipolar interaction between the two 'active' spins I and S. It has recently been shown [1,2] that long-lived states (LLS) may be excited by inducing a difference between the population of the singlet state $\mid S_{0}>$ and the average population of the three triplet states (vide infra). LLS are, to a very good approximation, immune to the mutual dipolar relaxation between the two 'active' spins, so that their life-times $T_{\text {LLS }}$ can in principle be indefinitely long, at any rate much longer than $T_{1}$. In practice, a factor $T_{\mathrm{LLS}} / T_{1}>37$ has been obtained for protons in favourable cases [3]. In nitrous oxide $\left(\mathrm{N}_{2} \mathrm{O}\right)$ enriched in ${ }^{15} \mathrm{~N}$, a LLS comprising two ${ }^{15} \mathrm{~N}$ nuclei can be sustained for several minutes [4]. Such long lifetimes of populations make it possible to extend the time scales over which phenomena such as slow exchange and slow translational diffusion may be characterised by NMR.

Extending the life-times of coherences will produce betterresolved spectra. Thus, by using spin echoes to achieve time-reversal $[5,6]$, one can reduce inhomogeneous broadening, thus revealing the underlying homogeneous widths, i.e., the line-widths that would be observed in a perfectly homogeneous field. Achieving further narrowing is a greater challenge. Homogeneous broadening results from transverse relaxation, which is due to stochastic interactions that cannot be eliminated by the experimentalist.

In some fortunate cases, mutual cancellation of these stochastic interactions can lead to line narrowing. For instance, if the interaction tensors have favourable relative orientations, the fluctuations of dipole-dipole interactions and anisotropic chemical shifts can partially cancel each other, a phenomenon which is used in Transverse Relaxation Optimised SpectroscopY (TROSY) [7-11]. Similar kinds of interferences between several dipolar interactions can also be observed in methyl groups [12]. These effects have brought major advances by increasing the size of proteins and protein complexes that are amenable to structural and dynamic studies by NMR.

This article shows how the use of coherent superpositions between singlet and triplet states, which we like to call long-lived coherences (LLCs) [13], can lead to a significant resolution enhancement in NMR spectroscopy. LLCs belong to the class of zero-quantum coherences (ZQCs). Transitions between singlet and triplet states, which belong to different irreducible representations of the permutation group, are normally symmetry-forbidden and therefore have vanishing transition probabilities. We shall see that under certain circumstances, it is easy to excite coherences across forbidden transitions. In the manner of two-dimensional (2D) spectroscopy, one can allow these coherences to undergo a precession under the effect of the Hamiltonian in a variable evolution interval $t_{1}$, prior to re-converting them into observable signals in a detection interval $t_{2}$.

Recently, Pileio, Carravetta and Levitt discussed similar transitions between symmetric and anti-symmetric states in a different context. In their ingenious experiments [14], the Zeeman polarised sample was removed adiabatically from the magnetic field. It is then possible to bring about a Rabi nutation of the two-level system comprising the singlet and central triplet states by irradiation with an electromagnetic field at extremely low frequency (ELF), typically on the order of a few tens of $\mathrm{Hz}$ [14]. It has been shown that this nutation can lead either to the inversion of the populations across the singlet-triplet transition or to the creation of a singlet-triplet coherence that oscillates at a frequency determined by the $J$-coupling constant.

There are several differences between 'ELF' and 'LLC' experiments. In the former case, the Hamiltonian is switched adiabatically [14], typically in the course of a few seconds, by shuttling the sample outside the $B_{0}$ field. The longitudinal relaxation may take its toll, particularly for protons in biological macromolecules, as evidenced by field-cycling 'relaxometry' experiments [15]. In ELF experiments, the desired coherence must then be excited by a slow Rabi nutation in low field, which in practice requires an ELF pulse on the order of a few seconds to obtain a nutation through an angle $\pi / 2$. By contrast, in our high-field LLC experiments, the spin system must be prepared by semi-selective inversion of one or more chosen multiplets in the proton spectrum. This requires one or several $r f$ pulses of few ms for a $\pi$ nutation angle, much shorter than $T_{1}$, although some losses due to $T_{2}$ relaxation during the pulses cannot be excluded. After semi-selective inversion, a continuous $r f$ field $B_{1}$ is suddenly switched on, superimposed on the high static $B_{0}$ field. This allows one to switch almost instantaneously (i.e., within a fraction of a $\mu$ s) to the desired Hamiltonian that is symmetrical under the spin permutation.

In contrast to the populations of long-lived states (LLS), which decay monoexponentially, the time-dependence of LLCs is oscillatory and must be mapped point-by-point in the manner of twodimensional (2D) spectroscopy. In NMR, the eigenbase can be changed simply by switching a radio-frequency $(r f)$ field on or off: if the $r f$ field is on, the states are either symmetric or antisymmetric under a permutation of the spins; if it is switched off, the states have no permutation symmetry because the difference between their Larmor precession frequencies robs the spins of their equivalence.

Like the popular TROSY method, the persistence of LLCs presupposes the existence of reasonably isolated scalar-coupled two-spin sub-systems. Unlike TROSY, however, isotope labelling of nitrogen15 or carbon-13 nuclei is not required for LLCs, and the beneficial effects do not require high static magnetic fields. The isolation of the sub-systems can be improved through partial deuteration. This can be achieved by procedures that are well known for biological molecules [12].

We have shown [13] that the lifetimes of LLCs are significantly less prone to homogeneous broadening than conventional singlequantum coherences. In addition - like other ZQCs - they are insensitive to inhomogeneous broadening. Therefore, LLC lifetimes can be significantly longer than the transverse relaxation times $T_{2}$ of single-quantum coherences that can be measured with spin-echo sequences, depending on the correlation time of rotational diffusion [16]. The extent of the resolution-enhancement effect can be described by the resolution-enhancement factor $\varepsilon=T_{\mathrm{LLC}} / T_{2}=\Delta v / \Delta v_{\mathrm{LLC}}$.

Predictions based on Redfield theory show that $3<\varepsilon<9$, where the lower limit $(\varepsilon=3)$ concerns small molecules in the extreme narrowing regime $\left(\tau_{\mathrm{c}} \ll 1 / \omega_{0}\right)$, and the upper limit $(\varepsilon=9)$ is relevant for slowly-tumbling macromolecules with $\tau_{\mathrm{c}} \gg 1 / \omega_{0}$ [16]. Slow oscillatory decays of LLCs, monitored by two-dimensional spectroscopy, lead to very narrow lines with half-widths at halfheight $\Delta v_{\mathrm{LLC}}=\left(\pi T_{\mathrm{LLC}}\right)^{-1} \ll \Delta v=\left(\pi T_{2}\right)^{-1}$. Here we report the observation of line-widths $\Delta v_{\mathrm{LLC}}=60 \mathrm{mHz}$ in 2,3,6-trichlorophenol in aqueous solution, where the resolution-enhancement factor is $\varepsilon \sim 2.4$. We have observed line-widths $\Delta v_{\mathrm{LLC}}=600 \mathrm{mHz}$ in ubiquitin [13] where the resolution-enhancement factor is $\varepsilon \sim 2.2$. In principle, if external relaxation mechanisms can be silenced, for example by replacing all protons in the vicinity by deuterons, a gain in line-width by a factor $\varepsilon \sim 9$ should be possible in macromolecules. In earlier work, we could only excite one LLC after the other in a selective fashion. Here, we show that we can also create and sustain several LLCs in parallel in different spin systems, such as different Gly residues in ubiquitin.

\section{The tailored Hamiltonian}

The Hamiltonian of a system comprising two coupled spins $\mathrm{I}=\mathrm{S}=1 / 2$ in a high magnetic field $B_{0}$, with chemical shifts $v_{\mathrm{I}, \mathrm{S}}=$ $-\gamma\left(1-\sigma_{\mathrm{I}, \mathrm{S}}\right) B_{0} /(2 \pi)$ and a scalar coupling constant $J$ can be expressed as usual in units of $h$ :

$H=v_{\mathrm{I}} I_{\mathrm{z}}+v_{\mathrm{S}} S_{\mathrm{z}}+\overrightarrow{J \mathbf{I}} \cdot \vec{S}$ 
Assuming without loss of generality that $v_{\mathrm{I}}>v_{\mathrm{S}}$, and defining $\Delta v_{\mathrm{IS}}=v_{\mathrm{I}}-v_{\mathrm{S}}>0$, the Hamiltonian can be truncated if the difference between the chemical shifts is much larger than the scalar coupling constant, $\Delta v_{\text {IS }} \gg|J|$,:

$H=v_{\mathrm{I}} I_{\mathrm{z}}+v_{\mathrm{S}} S_{\mathrm{z}}+J I_{\mathrm{z}} S_{\mathrm{z}}$

with an eigenbasis known as the product base (PB):

$\Phi_{\mathrm{PB}}=\{|\alpha \alpha\rangle,|\alpha \beta\rangle,|\beta \alpha\rangle,|\beta \beta\rangle\}=\left\{\left|\alpha_{\mathrm{I}} \alpha_{\mathrm{S}}\right\rangle,\left|\alpha_{\mathrm{I}} \beta_{\mathrm{S}}\right\rangle,\left|\beta_{\mathrm{I}} \alpha_{\mathrm{S}}\right\rangle,\left|\beta_{\mathrm{I}} \beta_{\mathrm{S}}\right\rangle\right\}$

where $\left|\alpha_{\mathrm{I}} \beta_{\mathrm{S}}\right\rangle=\left|m_{\mathrm{I}}=+1 / 2, m_{\mathrm{S}}=-1 / 2\right\rangle$ etc. If the static field vanishes $\left(B_{0}=0\right)$, i.e., if the sample is removed from the field, the two spins I and $S$ become magnetically equivalent:

$H=J \overrightarrow{\mathrm{I}} \cdot \vec{S}$

The eigenbasis of this Hamiltonian, which is known as the singlet-triplet base (STB), is appropriate to describe a pair of equivalent spins comprising three symmetric triplet states and one anti-symmetric singlet state:

$\Phi_{\text {STB }}=\left\{\left|T_{+1}\right\rangle,\left|T_{0}\right\rangle,\left|S_{0}\right\rangle,\left|T_{-1}\right\rangle\right\}$

where

$T_{+1}=|\alpha \alpha\rangle ; T_{0}=N(|\alpha \beta\rangle+|\beta \alpha\rangle) ; S_{0}=N(|\alpha \beta\rangle-|\beta \alpha\rangle) ; T_{-1}=|\beta \beta\rangle$

and $N=2^{-1 / 2}$

The two spins I and S become magnetically equivalent if a continuous-wave (CW) radio-frequency $(r f)$ with an amplitude $\left|v_{1}\right|=\left|-\gamma B_{1} /(2 \pi)\right| \gg \Delta v_{\mathrm{IS}}$ is applied and the $r f$ carrier $v_{\mathrm{RF}}$ is placed half-way between the two chemical shifts,

$v_{\mathrm{RF}}=v_{\mathrm{av}}=\left(v_{\mathrm{I}}+v_{\mathrm{S}}\right) / 2$.

A frame rotating at $v_{\mathrm{av}}$ leads to the Hamiltonian:

$H=v_{1}\left(I_{\mathrm{x}}+S_{\mathrm{x}}\right)+J \overrightarrow{\mathrm{I}} \cdot \overrightarrow{\mathrm{S}}+\frac{\Delta v_{\mathrm{IS}}}{2}\left(I_{\mathrm{z}}-S_{\mathrm{z}}\right)$.

The last term involves $\left(I_{z}-S_{z}\right)$, which does not commute with $\left(I_{\mathrm{x}}+S_{\mathrm{x}}\right)$ and can be neglected when $v_{1} \gg \Delta v_{\mathrm{IS}}$ :

$H=v_{1}\left(I_{\mathrm{x}}+S_{\mathrm{x}}\right)+\vec{J} \overrightarrow{\mathrm{I}} \cdot \vec{S}$.

This Hamiltonian is obviously symmetric under a permutation of the spins I and $S$. Its basis, STB, is given by Eq. (5).

The conversion $\Phi_{\mathrm{PB}}=V \cdot \Phi_{\mathrm{STB}}$ is described by the matrix:

$V=\left(\begin{array}{cccc}1 & 0 & 0 & 0 \\ 0 & N & N & 0 \\ 0 & N & -N & 0 \\ 0 & 0 & 0 & 1\end{array}\right)$,

with $V=V^{-1}$ and $N=2^{-1 / 2}$. Products of Cartesian components of angular momentum operators ('product operators') can provide a convenient description of coherence transfer processes. They can be transformed from the PB into the STB:

$B_{\mathrm{PB}}=V^{-1} B_{\mathrm{STB}} V$.

The effective fields experienced by the spins I and $S$ are tilted through angles $\theta_{\mathrm{I}, \mathrm{S}}$ with respect to the $z$ axis if an $r f$ field $B_{1}$ is applied at $v_{\mathrm{av}}$, exactly half-way between the two chemical shifts:

$\tan \theta_{\mathrm{I}, \mathrm{S}}=\frac{ \pm 2\left|v_{1}\right|}{\Delta v_{\mathrm{IS}}}$

with $0<\theta_{\mathrm{I}, \mathrm{S}}<\pi$. If $v_{1} \gg \Delta v_{\mathrm{IS}}, \theta_{\mathrm{I}, \mathrm{S}} \sim \pi / 2$, hence $\sin \theta_{\mathrm{I}, \mathrm{S}} \approx 1$ and $\cos \theta_{\mathrm{I}, \mathrm{S}} \approx 0$.

When a continuous radio-frequency field $B_{1}$ is suddenly applied along the $x$-axis of the rotating frame, the eigenstates of the system change instantaneously from the product base $\Phi_{\mathrm{PB}}$ to the singlet-triplet base $\Phi_{\mathrm{STB}}$. In the presence of an $r f$ field, the operators of spins $K=\{\mathrm{I}, \mathrm{S}\}$ are transformed into the tilted interaction frame by:

$$
\left(\begin{array}{c}
K_{\mathrm{x}}^{\prime} \\
K_{\mathrm{y}}^{\prime} \\
K_{\mathrm{z}}^{\prime}
\end{array}\right)=\left(\begin{array}{ccc}
\cos \theta_{\mathrm{k}} & 0 & -\sin \theta_{\mathrm{k}} \\
0 & 1 & 0 \\
\sin \theta_{\mathrm{k}} & 0 & \cos \theta_{\mathrm{k}}
\end{array}\right)\left(\begin{array}{c}
K_{\mathrm{x}} \\
K_{\mathrm{y}} \\
K_{\mathrm{z}}
\end{array}\right)
$$

When $\theta_{\mathrm{I}, \mathrm{S}} \sim \pi / 2$, this simply amounts to relabeling $K_{\mathrm{z}}^{\prime} \rightarrow K_{\mathrm{x}}$.

\section{Definition of long-lived coherences}

A coherent superposition between the singlet and central triplet states, which we call $Q_{\mathrm{LLC}}$, can be readily defined in the STB $[3,13]$ :

$Q_{\mathrm{LLC}}=\left|S_{0}\right\rangle\left\langle T_{0}|+| T_{0}\right\rangle\left\langle S_{0}\right|$

This can be converted into Cartesian angular momentum operators that are more familiar in the field of NMR [17]:

$Q_{\mathrm{LLC}}=I_{\mathrm{X}}-S_{\mathrm{X}}$

Thus, when the continuous $r f$ field is suddenly switched on, the difference $\left(I_{\mathrm{x}}-S_{\mathrm{x}}\right)$ in the product base $\Phi_{\mathrm{PB}}$ is converted into offdiagonal elements between the states $\left|S_{0}\right\rangle$ and $\left|T_{0}\right\rangle$ in the singlet-triplet base $\Phi_{\text {STB }}$ (Table A2 in Appendix). These off-diagonal elements correspond to long-lived coherences (LLCs). In Table A3 (Appendix), it is shown how the matrix representations of a few populations and coherences in the singlet-triplet base $\Phi_{\text {Ств }}$ can be expressed in the product base $\Phi_{\mathrm{PB}}$.

A density operator of the form $Q_{\mathrm{LLC}}$ in Eq. (15) commutes with the first term of the Hamiltonian in Eq. (9), but the second isotropic scalar coupling term causes it to undergo an evolution:

$\left[I_{\mathrm{x}}-S_{\mathrm{x}}, \overrightarrow{\mathrm{I}} \cdot \vec{S}\right]=i\left(2 I_{\mathrm{y}} S_{\mathrm{z}}-2 I_{\mathrm{z}} S_{\mathrm{y}}\right)$

Solving the Liouville-von Neumann equation [17] (without considering relaxation for the time being) allows the evolution of the LLC to be described, provided that the applied $r f$ field has an amplitude that is much larger than the chemical shift difference of the two spins:

$Q_{\mathrm{LLC}}(t)=\left(I_{\mathrm{x}}-S_{\mathrm{x}}\right) \cos (2 \pi J t)+\left(2 I_{\mathrm{y}} S_{\mathrm{z}}-2 I_{\mathrm{z}} S_{\mathrm{y}}\right) \sin (2 \pi J t)$

Thus, the LLC oscillates at a frequency given by the scalar coupling.

\section{Relaxation of long-lived coherences}

In addition to the coherent effect of the scalar spin-spin interaction, the LLC decays through relaxation,

$\frac{d}{d t} Q_{\mathrm{LLC}}=-\left(R_{\mathrm{LLC}}+i 2 \pi J\right) Q_{\mathrm{LLC}}$

The LLC oscillates with a frequency determined by the scalar coupling constant $J$ (imaginary part), while the rate $R_{\mathrm{LLC}}$ (real part) governs the decay. The oscillation frequency of these coherences is independent of the chemical shifts, and, by extension, of the local value of the $B_{0}$ field, so that LLCs, like other zeroquantum coherences (ZQCs), are insensitive to inhomogeneous broadening. The lifetime $T_{\mathrm{LLC}}=1 / R_{\mathrm{LLC}}$ can be significantly longer than the transverse relaxation times $T_{2}=1 / R_{2}$ of single-quantum coherences. In a system of two spin- $1 / 2$ nuclei $I$ and $S$, the rate $R_{2}$ is predominantly determined by the dipolar interaction between these spins [18]:

$R_{2}=\left(\frac{b_{\mathrm{IS}}^{2}}{20}\right)[9 J(0)+15 J(\omega)+6 J(2 \omega)]+R_{2}^{e x t}$

where $b_{\mathrm{IS}}=-\frac{\mu_{0}}{4 \pi} \frac{\gamma^{2} h}{r_{\mathrm{IS}}^{3}}$ is the strength of the homonuclear dipolar coupling, $r_{\mathrm{IS}}$ the distance between the spins I and $\mathrm{S}, J(\omega)=\frac{\tau_{\mathrm{c}}}{1+\omega^{2} \tau_{\mathrm{c}}^{2}}$ the 
spectral density function, i.e., the Fourier transform of the autocorrelation function that describes the rotational diffusion of the molecule, $\tau_{\mathrm{c}}$ the rotational correlation time, and the other quantities have their usual meaning. The term $R_{2}^{\text {ext }}$ comprises contributions from external random fields, anisotropic chemical shifts (CSA), and dipole-dipole (DD) interactions to remote spins.

In contrast to 'longitudinal' LLS, which are not affected by the dipolar interaction between the two spins I and S, the 'transverse' relaxation rate constant $R_{\mathrm{LLC}}$ under the homonuclear dipolar interaction is given by [13]:

$R_{\mathrm{LLC}}=\left(\frac{b_{\mathrm{IS}}^{2}}{20}\right)[J(0)+3 J(\omega)+6 J(2 \omega)]+R_{\mathrm{LLC}}^{\text {ext }}$

If the $R_{\mathrm{LLC}}^{\text {ext }}$ term is small, the LLC has a slower relaxation rate than single-quantum coherences in all motional regimes, i.e., for arbitrary molecular tumbling rates. The ratio is $T_{\mathrm{LLC}} / T_{2}=\varepsilon=3$ in the fast-tumbling limit $\left(\tau_{\mathrm{c}} \ll 1 / \omega_{0}\right)$, when the spectral densities are equal at all frequencies. This ratio increases to $\varepsilon=9$ for slow tumbling $\left(\tau_{\mathrm{c}} \gg 1 / \omega_{0}\right)$. Thus, if the $R_{\mathrm{LLC}}^{\text {ext }}$ terms may be neglected, the ratio of the transverse relaxation times should increase from $T_{\mathrm{LLC}} / T_{2}=\varepsilon=3$ in small molecules to $T_{\mathrm{LLC}} / T_{2}=\varepsilon=9$ in macromolecules, thus making the gain more significant for large molecules.

\section{Designing suitable experiments}

A pulse sequence suitable for exciting and detecting LLCs [13] is shown in Fig. 1. We shall provide a step-by-step description of the method.

In the beginning of the experiment, at point $\tau_{(1)}$ in the sequence, the density operator $\sigma_{1}$ comprises only populations in thermal equilibrium, conveniently described by the operators $I_{z}$ and $S_{z}$ in Table A1 (Appendix):

$\sigma_{1}=E / 2-a\left(I_{z}+S_{z}\right)-b\left(I_{z}-S_{z}\right)$

where $a=h v_{\mathrm{av}} /\left(4 k_{\mathrm{B}} T\right)$ and $b=h \Delta v_{\mathrm{IS}} /\left(8 k_{\mathrm{B}} T\right)$ in the high-temperature limit, where the Boltzmann distribution is linear in $1 / T$. Since the unity operator, $E$, does not affect the outcome of NMR experiments if thermal correction factors can be neglected [19], and since $b \ll a$, we set $a=1$ for simplicity and truncate as usual:

$\sigma_{1}=\left(I_{z}+S_{z}\right)$.

A semi-selective $(\pi)_{\mathrm{x}}$ pulse is applied only to spin $S$ between $\tau_{(1)}$ and $\tau_{(2)}$ :

$R_{(1->2)}=\exp \left(-i \pi S_{\mathrm{x}}\right)$

so that the system at point $\tau_{(2)}$ is described by:

$\sigma_{2}=R_{(1->2)} \sigma_{1} R_{(1->2)}^{-1}=I_{z}-S_{z}$.

After applying a non-selective $(\pi / 2)$ y pulse to both spins:

$R_{(2->3)}=\exp \left[-i \frac{\pi}{2}\left(I_{\mathrm{y}}+S_{\mathrm{y}}\right)\right]$

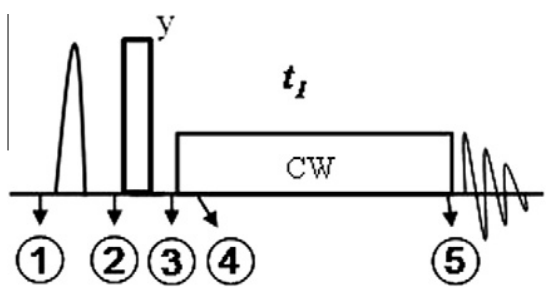

Fig. 1. Basic experiment used for excitation, sustaining and detection of long-lived coherences (LLCS) for one pair of spins at a time. The numbers in circles are mentioned in the equations. The open rectangle represents a non-selective $\pi / 2$ pulse. All pulses are applied along the $x$ axis. A typical $\pi$ pulse for semi-selective inversion has a sinc shape, a length of $70 \mathrm{~ms}$, and a peak $r f$ amplitude of $40 \mathrm{~Hz}$ one obtains:

$\sigma_{3}=R_{(2->3)} \sigma_{2} R_{(2->3)}^{-1}=I_{\mathrm{x}}-S_{\mathrm{x}}$

Between points $\tau_{(3)}$ and $\tau_{(4)}$, a CW rf field is switched on, so that the eigenstates of the system are switched instantaneously to the symmetry-adapted states $\Phi_{\text {STB }}$. As explained in Eq. (13), we can now rewrite the density operator during $\tau_{(4)}$, since $\theta_{\mathrm{I}, \mathrm{S}} \sim \pi / 2$ :

$\sigma_{4}=I_{\mathrm{x}}-S_{\mathrm{x}}=I_{\mathrm{z}}^{\prime}-S_{\mathrm{z}}^{\prime}$.

Table A2 shows how the matrix representation of the density operator $\sigma_{4}$ in the product base $\Phi_{\mathrm{PB}}$ can be transformed into the singlet-triplet base $\Phi_{\mathrm{STB}}$ :

$\sigma_{4}=I_{\mathrm{z}}^{\prime}-S_{\mathrm{z}}^{\prime}=\left|S_{0}><T_{0}\right|+\left|T_{0}><S_{0}\right|$.

\section{Long-lived coherences in a small molecule}

In 2,3,6-trichlorophenol in a field $B_{0}=9.4 \mathrm{~T}(400 \mathrm{MHz}$ for protons) at room temperature, a ratio $\varepsilon=T_{\mathrm{LLC}} / T_{2} \sim 2.4$ has been observed, i.e., a resolution enhancement by a factor 2.4. It follows from Eq. (20) that $\varepsilon$ is attenuated by uncorrelated external contributions to the relaxation rates. In analogy to relaxation rates $R_{\mathrm{LLS}}$ of long-lived states, which are immune to fluctuations associated with CSA and DD tensors that are symmetrical under permutation of spins I and S [20,21], the lifetimes of long-lived coherences will be affected to a lesser extent by spin-permutation symmetric relaxation mechanisms.

The coherent and relaxation properties of LLCs lead to 2D spectra with very high resolution, which can be used either to resolve nearlydegenerate signals or to measure $J$-couplings (and residual dipolar splittings in weakly oriented phases) with an unprecedented
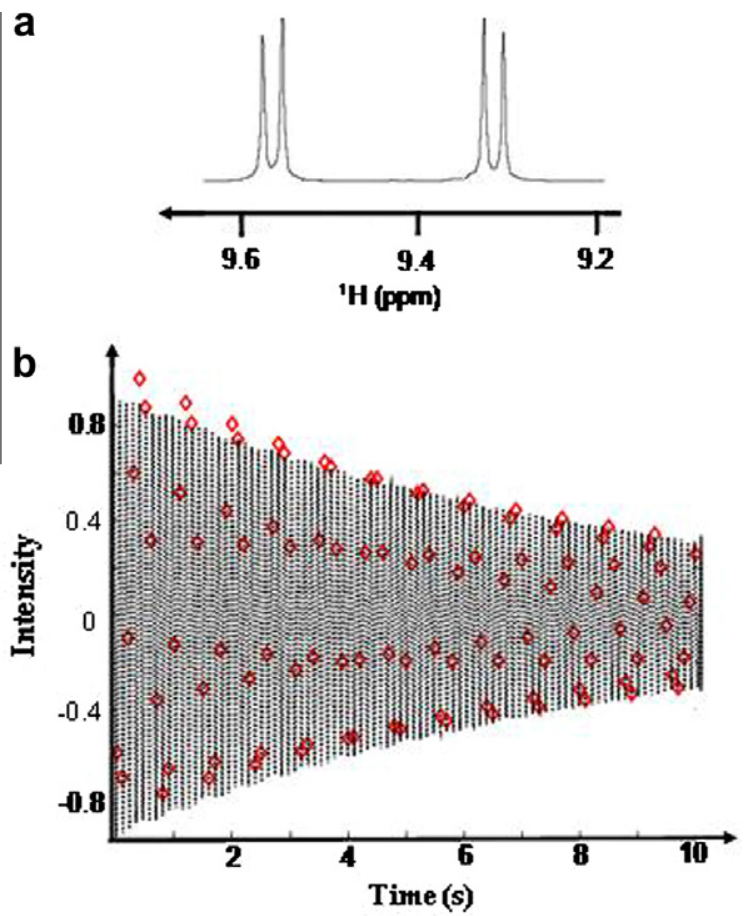

Fig. 2. (a) Proton spectrum of the two weakly coupled protons in 2,3,6-trichlorophenol at $400 \mathrm{MHz}$. (b) The experimental intensities (red lozenges) were plotted as a function of time $t_{1}$ and fitted with a function $I\left(t_{1}\right)=I_{0} \cos \left(2 \pi J t_{1}\right) \exp \left(-R_{\mathrm{LLC}} t_{1}\right)$. The best-fit parameters were $J=8.760 \pm 0.001 \mathrm{~Hz}$ and $R_{\mathrm{LLC}}=0.10 \pm 0.01 \mathrm{~s}^{-1}$. The amplitude of the continuous rf irradiation was $2.5 \mathrm{kHz}$. Each experiment was performed with four transients with a relaxation delay of $30 \mathrm{~s}$. $J=8.8 \mathrm{~Hz}$ was obtained from a conventional 1D spectrum (For interpretation of the references to colour in this figure legend, the reader is referred to the web version of this article). 

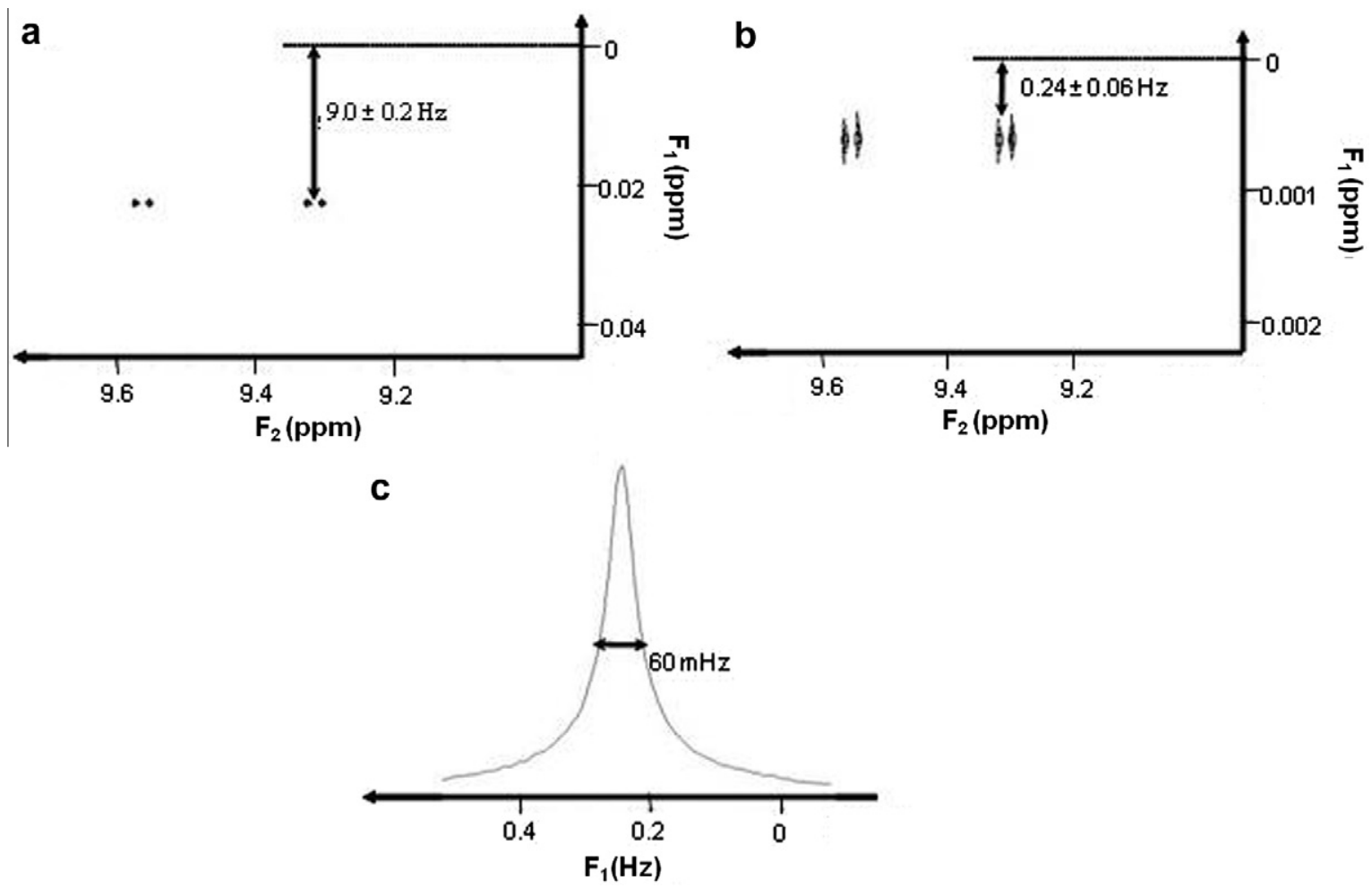

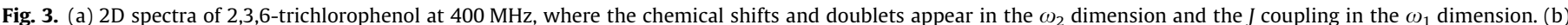

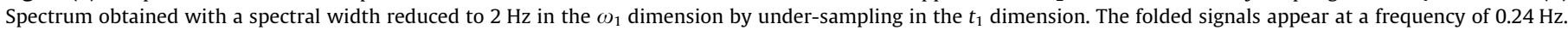

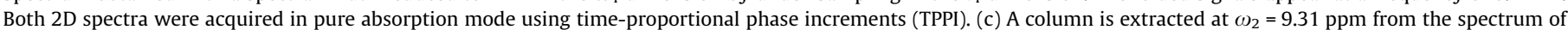
Fig. 3b. The linewidth is merely $60 \mathrm{mHz}$ in the $\omega_{1}$ dimension.

accuracy in high field, albeit less accurately than in zero field [14]. The experiments were performed with the pulse sequence of Fig. 1. Fig. 2 shows the typical spectrum of two coupled spins- $1 / 2$, as well as the time-domain decay of LLCs in 2,3,6-trichlorophenol. In the 2D spectrum in Fig. 3a the chemical shifts are seen in the direct $\omega_{2}$ dimension, whereas only a single peak at $9.0 \pm 0.2 \mathrm{~Hz}$ is observed in the $\omega_{1}$ dimension. This corresponds to the magnitude of the coupling constant $J$. Here, the $\omega_{1}$ dimension was not sampled long enough to avoid truncation effects $\left(t_{1}^{\max }=14.3 \mathrm{~s}\right.$ with 1024 points and $\Delta t_{1}=14 \mathrm{~ms}$ ). To obtain the linewidth permitted by this method, one has to sample the indirect dimension as far as $t_{1}^{\max }=5 T_{\mathrm{LLC}}$. The $2 \mathrm{D}$ spectrum in Fig. 3b was recorded by under-sampling the indirect dimension $\left(t_{1}^{\mathrm{max}}=50 \mathrm{~s}\right.$ with $N_{1}=200$ points and $\left.\Delta t_{1}=250 \mathrm{~ms}\right)$. The linewidth is only $60 \mathrm{mHz}$, unaffected by truncation. The spectral width was reduced to $2 \mathrm{~Hz}$ in the indirect dimension, so that the signal appeared at the (aliased) frequency $v_{1}=0.24 \mathrm{~Hz}$. The true frequency $v_{1}=J=8.760 \pm 0.001 \mathrm{~Hz}$ can be found by adding 4.5 times the spectral width.

\section{Long-lived coherences in a protein}

In the terminal residue Gly-76 of ubiquitin it was found that $\varepsilon=T_{\mathrm{LLC}} / T_{2}=2.2$ [13]. The 2D experiment performed with the sequence of Fig. 1 in ubiquitin reveals chemical shifts and doublets in the $\omega_{2}$ dimension, whereas only one frequency, which corresponds to the aliased $J$ coupling, is detected in the $\omega_{1}$ dimension. Under-sampling leads to spectral aliasing, but it is easy to correct for this effect and find that the aliased signal corresponds to $J=-17.17 \mathrm{~Hz}$. The full line-width at half-height of the signal was $\Delta v_{1 / 2}=600 \mathrm{mHz}$ in the $\omega_{1}$ dimension, whereas the homogeneous linewidths of single-quantum coherences, estimated from a separate spin-locking $R_{1}$-experiment, are $\Delta v_{1 / 2} \sim 1.4 \mathrm{~Hz}$.

\section{Simultaneous excitation of several long-lived coherences}

LLC experiments are not limited to studying one pair of spins after another. The semi-selective inversion of several spins in rapid succession (i.e., within an interval shorter than $T_{1}$ ) allows several LLCs to be excited simultaneously in different sub-systems. Here we demonstrate that LLCs can be excited simultaneously for the two C-terminal residues Gly-75 and Gly-76 in ubiquitin. We have designed the pulse sequence shown in Fig. 4a to achieve this. It is to be noted that there are three important differences between the simultaneous selection and selective methods for detecting LLCs. First, we have inserted a double-quantum (DQ) filter. Second, we need two semi-selective $\pi$ pulses to invert two doublets. Third, the $r f$ carrier was placed at the mean position between the two averages $v_{\mathrm{av}}(I, S)$ and $v_{\mathrm{av}}\left(I^{\prime}, S^{\prime}\right)$ of the four chemical shifts during the sustaining period. We used a train of sinc shaped pulses [22] to sustain the LLCs on both residues simultaneously because the use of a simple CW rf is not sufficient in this case. The oscillation frequency is not exactly equal to the scalar coupling, as the condition leading from Eq. (8) to Eq. (9) is not fully satisfied. However, the unaccounted coherent evolution terms in the Hamiltonian have a very limited effect $(<1 \%$, vide infra) in the precession rate of the coherences.

There are some new elements in Fig. 4a with respect to Fig. 1. Presaturation by a continuous-wave $r f$ field ('CW' in Fig. 4a) was used to attenuate the water signal. A $D Q$ filter was used to suppress perturbing multiplets with smaller splittings that arise from other spin systems in the protein. This filter consisted of a sequence of pulses, phase cycling to select the double-quantum coherence, pulsed field gradients, and matched delays $\tau=1 /(4 J)=14.7 \mathrm{~ms}$, since $|J|=17 \mathrm{~Hz}$ for all glycine residues (irrespective of their environment). This filter creates pure in-phase coherences of the form $\left(I_{\mathrm{x}}+S_{\mathrm{x}}\right)$ for Gly-75 and Gly-76. Note that this filter also suppresses 

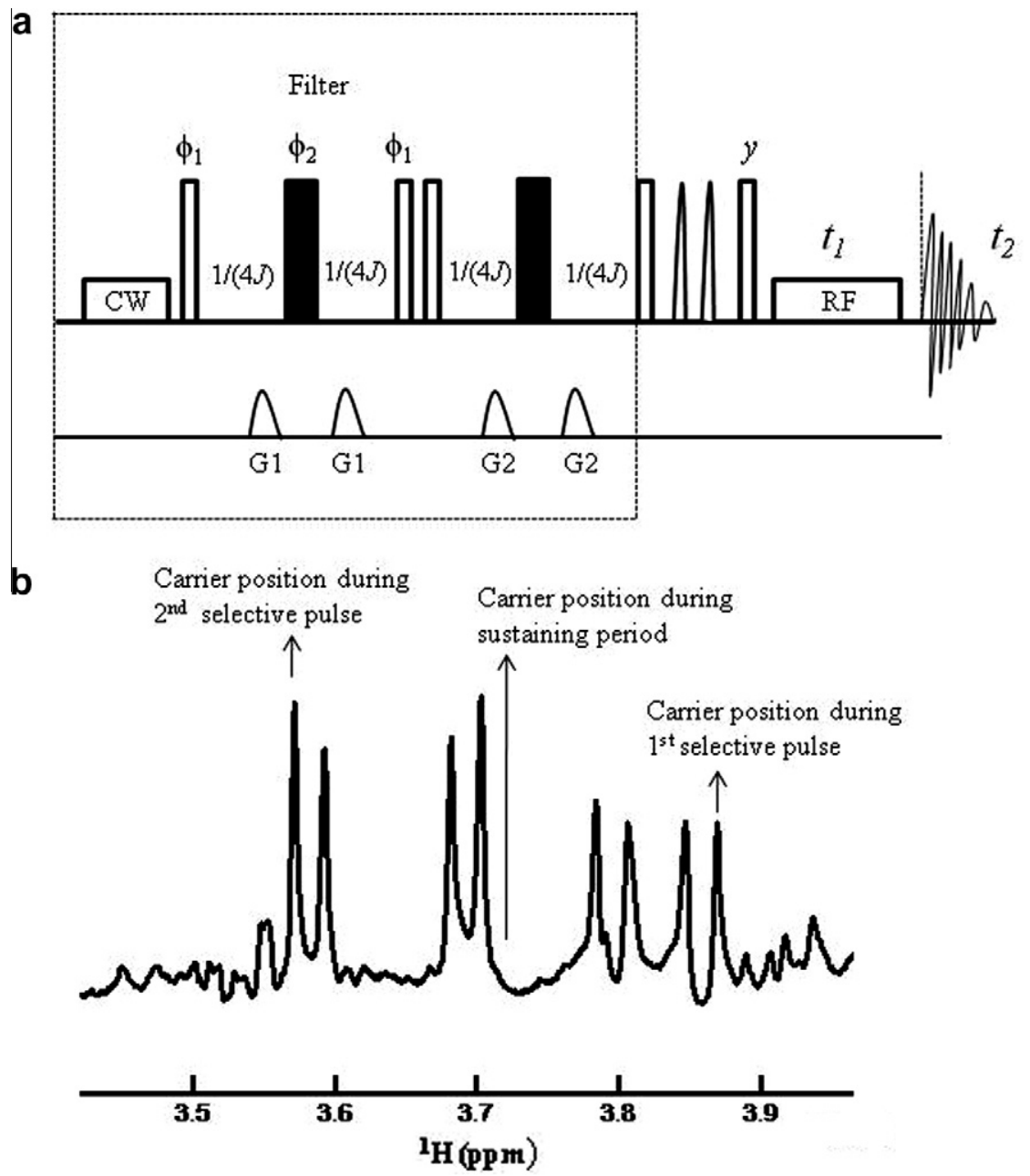

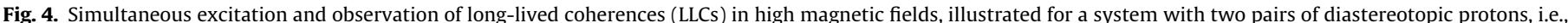

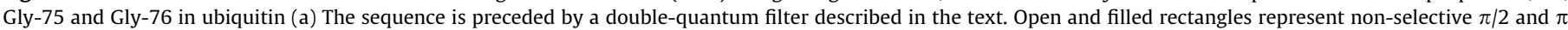

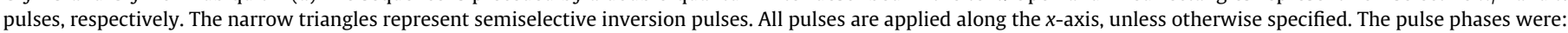

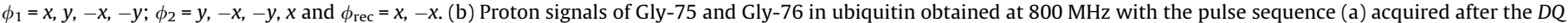
filter. The positions of the $r f$ carrier at different time points are shown.

singlets quite efficiently. If no filters are used, the baseline of the LLC experiment can be contaminated by unwanted signals from residues other than glycines, which degrade the signal purity and render the fitting process inaccurate.

Fig. 5 shows signals of Gly-76 and Gly-75 in ubiquitin excited and observed with the simultaneous excitation method. Fitting yields modulation frequencies (which are not identical to the scalar coupling constants J, vide supra) $v_{1}=17.20 \pm 0.01 \mathrm{~Hz}$ for Gly-76 and $J=16.80 \pm 0.05 \mathrm{~Hz}$ for Gly-75. The relaxation rates were found to be $R_{\mathrm{LLC}}=2.1 \pm 0.1 \mathrm{~s}^{-1}$ for Gly-76 and $2.1 \pm 0.2 \mathrm{~s}^{-1}$ for Gly-75. These results were compared against standard, narrow-band LLC experiments in the manner of Fig. 1, performed separately for Gly-76 and Gly-75. The agreement between the two methods was found to be satisfactory. The $2 \mathrm{D}$ version of this LLC experiment is shown in Fig. $5 b$.

\section{Conclusions}

Remarkably narrow lines can be obtained in solution-state NMR spectra in high fields by exploiting the long life-times associated with superpositions of quantum states with different symmetry, known as long-lived coherences (LLCs). These can be compared with coherences that can be excited by extremely low frequency (ELF) irradiation in a vanishing static field. In high fields, LLCs can be excited almost instantaneously by spin-locking after a semi-selective population inversion. LLCs may be regarded as a special class of zero-quantum coherences (ZQCs). The slow oscillatory decays of long-lived coherences, which are characterised by decay constants $T_{\mathrm{LLC}}$ that can be on the order of $5 \mathrm{~s}$ in our examples, yield spectra with line-widths $\Delta v_{\mathrm{LLC}}$ on the order of $60 \mathrm{mHz}$. Scalar coupling constants and residual dipolar couplings (RDCs) can be, therefore, determined with unprecedented accuracy. The slow decay rates are very sensitive to extraneous relaxation mechanisms such as those arising from dilute paramagnetic impurities. In small molecules in the extreme narrowing limit, the line-widths $\Delta v_{\mathrm{LLC}}$ can be a factor 3 narrower than in spectra of ordinary single-quantum coherences (SQCs), provided relaxation is driven only by the dipole-dipole interaction between the two spins that participate in the LLC. In the slow motion limit for large molecules, the improvement in line-widths can in principle be a factor 9 in favourable cases, provided that extraneous relaxation mechanisms do not operate. This opens perspectives of studying molecular assemblies that are almost an order of magnitude larger than was hitherto possible. Generalisations to various other forms of spectroscopy other than NMR are conceivable if some means can 


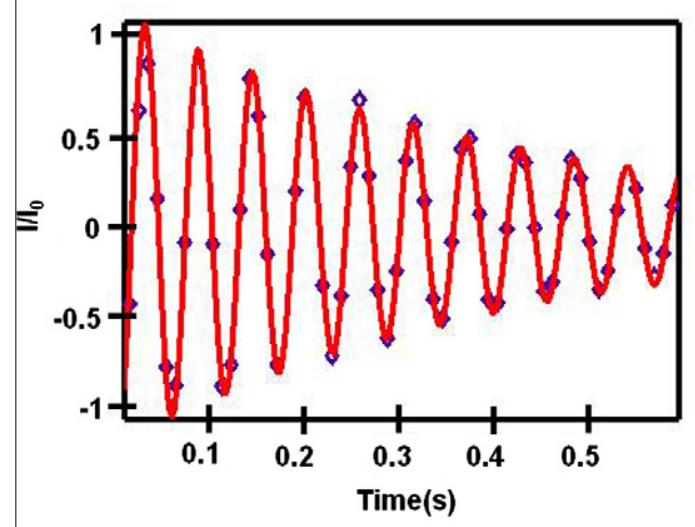

a
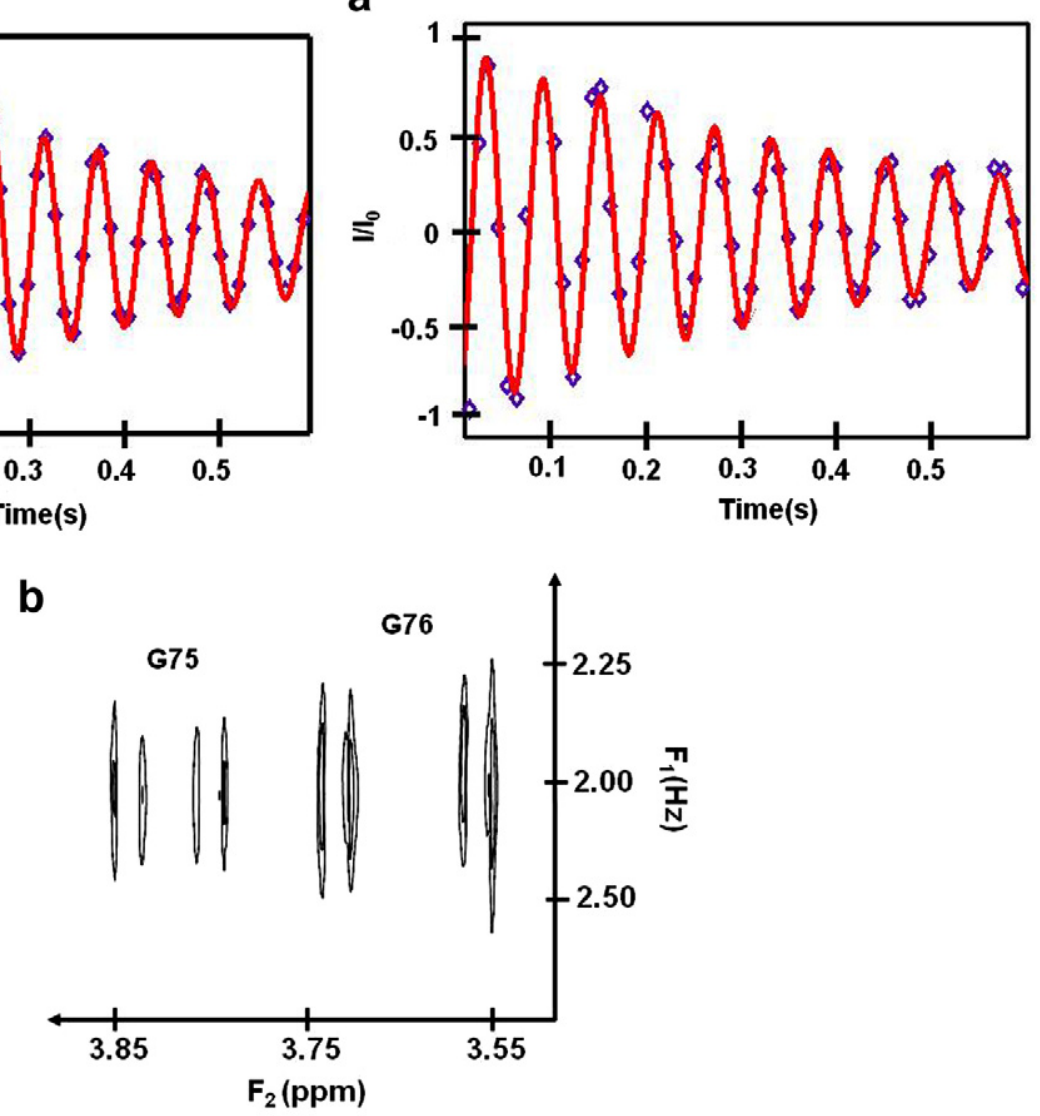

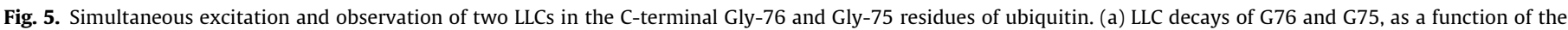

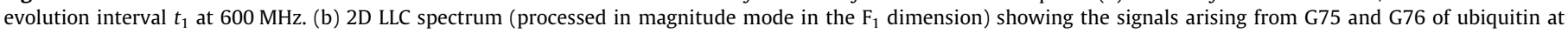

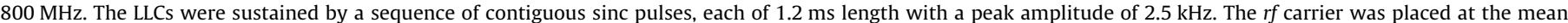

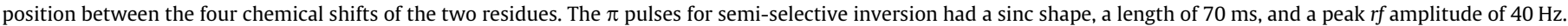

\section{Table A1}

A selection of matrix representations of products of angular momentum operators for spin-1/2 nuclei in a two-spin system, expressed in the product base $\Phi_{\mathrm{PB}}$ and in the singlet-triplet base $\Phi_{\text {STB }}$.

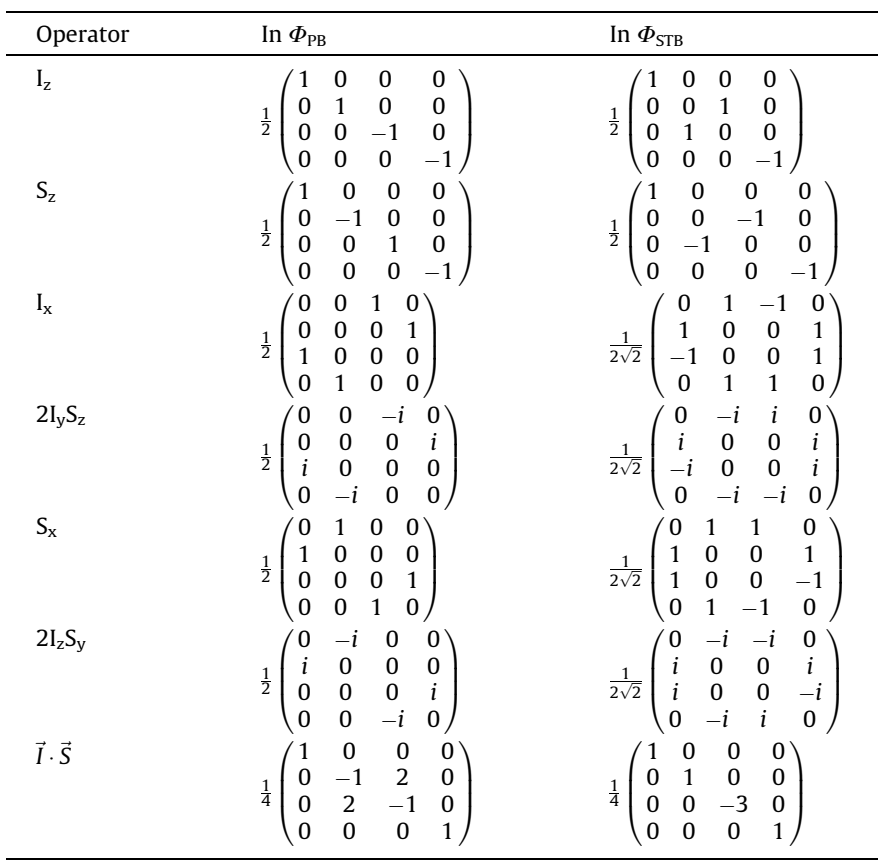

be found to switch between symmetrical and non-symmetrical Hamiltonians.

\section{Acknowledgements}

The authors thank Malcolm Levitt (University of Southampton) for numerous discussions. Martial Rey provided valuable technical assistance. This work was supported by the Swiss National Science Foundation ('Ambizione' grant PZOOP2_121928 to P.V. and FNRS grant 200020_124694 to G. B. and P. V.), the Ecole Polytechnique Fédérale de Lausanne (EPFL), the Swiss Commission for Technology and Innovation (CTI grant 9991.1 PFIW-IW to G. B., Paul Dyson, Jean-Philippe Ansermet, and P. V.), and the French CNRS. P.V. thanks the Faculty of Basic Sciences, EPFL, for financial support.

\section{Appendix A}

To construct the matrix representations of products of angular momentum operators, we start with the Pauli matrices for an isolated spin $K=1 / 2$, supplemented by the identity operator $E_{2}$ :

$K_{x}=\frac{1}{2}\left(\begin{array}{cc}0 & 1 \\ 1 & 0\end{array}\right) ; K_{y}=\frac{i}{2}\left(\begin{array}{cc}0 & -1 \\ 1 & 0\end{array}\right) ; K_{z}=\frac{1}{2}\left(\begin{array}{cc}1 & 0 \\ 0 & -1\end{array}\right) ; E_{2}=\left(\begin{array}{ll}1 & 0 \\ 0 & 1\end{array}\right)$

For a system with two spins $I=1 / 2$ and $S=1 / 2$, one can readily construct a basis consisting of matrix representations of 16 normalized products of angular momentum operators: 
Table A2

Transformation of the difference $I_{\mathrm{x}}-S_{\mathrm{x}}$ in the product base $\Phi_{\mathrm{PB}}$ into the singlet-triplet base $\Phi_{\mathrm{STB}}$ when the $r f$ field is switched on. The two off-diagonal elements in $\Phi_{\text {STB }}$ correspond to long-lived coherences. The basis kets are in the same order as in Eqs. (3) and (5).

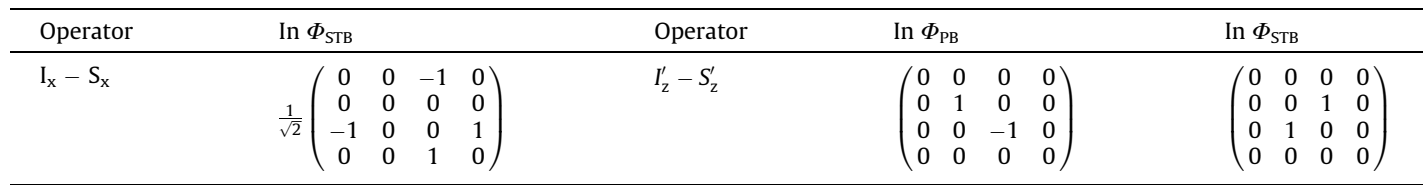

Table A3

A selection of matrix representations of operators: diagonal elements represent populations and off-diagonal elements ordinary coherences in the product base $\Phi_{\mathrm{PB}}$ or long-lived coherences (LLC) in the singlet-triplet base $\Phi_{\text {STB }}$.

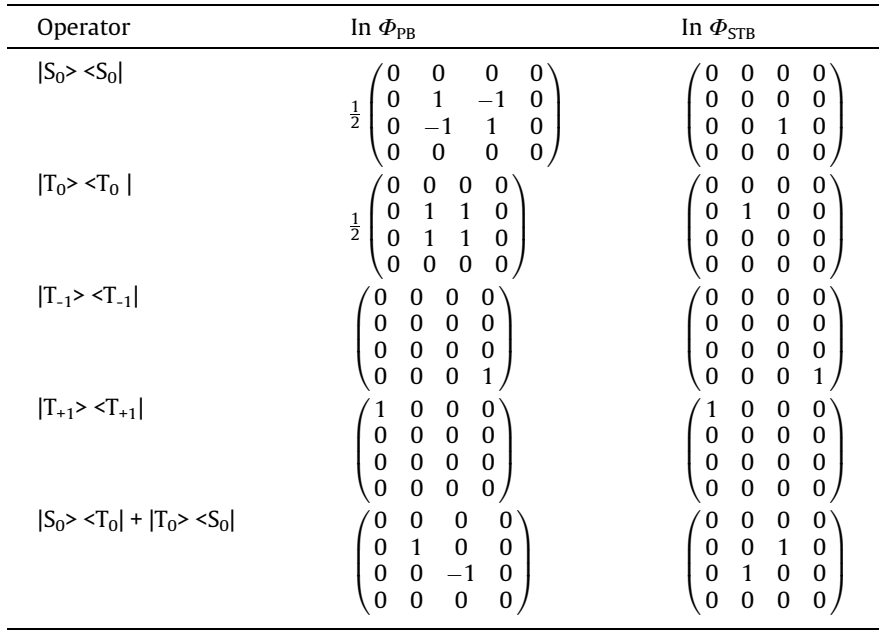

$E=\frac{1}{2} E_{2} \otimes E_{2}$

$I_{\mu}=K_{\mu} \otimes E_{2}$

$S_{\mu}=E_{2} \otimes K_{\mu}$

$2 I_{\mu} S_{v}=2 K_{\mu} \otimes K_{v}$,

where, $\mu, v=\{x, y, z\}$. Some examples of product operators that are relevant for this work are shown in Table A1, both in the usual product base $\Phi_{\mathrm{PB}}$ and in the singlet-triplet base $\Phi_{\mathrm{STB}}$.

See Tables A1-A3.

\section{References}

[1] M. Carravetta, O.G. Johannessen, M.H. Levitt, Beyond the $T_{1}$ limit: singlet nuclear spin states in low magnetic fields, Phys. Rev. Lett. 92 (2004) 153003.
[2] M. Carravetta, M.H. Levitt, Long-lived nuclear spin states in high-field solution NMR, J. Am. Chem. Soc. 126 (2004) 6228-6229.

[3] R. Sarkar, P.R. Vasos, G. Bodenhausen, Singlet-state exchange NMR spectroscopy for the study of very slow dynamic processes, J. Am. Chem. Soc. 129 (2007) 328-334.

[4] G. Pileio, M. Carravetta, E. Hughes, M.H. Levitt, The long-lived nuclear singlet state of N-15-nitrous oxide in solution, J. Am. Chem. Soc. 130 (2008) 12582 12583.

[5] P. Pelupessy, E. Rennella, G. Bodenhausen, High-resolution NMR with unknown spatiotemporal variations of magnetic fields, Science 324 (2009) 1693-1697.

[6] C.A. Meriles, D. Sakellariou, H. Heise, A.J. Moule, A. Pines, Approach to highresolution ex situ NMR spectroscopy, Science 293 (2001) 82-85.

[7] M. Goldman, Interference effects in the relaxation of a pair of unlike spin- $1 / 2$ nuclei, J. Magn. Reson. 60 (1984) 437-452.

[8] H. Shimizu, Theory of the dependence of nuclear magnetic relaxation on the absolute sign of spin-spin coupling constant, J. Chem. Phys. 40 (1964) 3357.

[9] E.L. Mackor, C. MacLean, Sign of $\mathrm{J}_{\mathrm{HF}}$ in $\mathrm{CHFCl}_{2}$, J. Chem. Phys. 44 (1966) 64.

[10] S. Wimperis, G. Bodenhausen, Relaxation-allowed cross-peaks in twodimensional NMR correlation spectroscopy, Mol. Phys. 66 (1989) 897-919.

[11] S. Wimperis, G. Bodenhausen, Relaxation-allowed transfer of coherence in NMR between spins which are not scalar coupled, Chem. Phys. Lett. 140 (1987) 41-45.

[12] V. Tugarinov, P.M. Hwang, J.E. Ollerenshaw, L.E. Kay, Cross-correlated relaxation enhanced $\mathrm{H}-1-\mathrm{C}-13$ NMR spectroscopy of methyl groups in very high molecular weight proteins and protein complexes, J. Am. Chem. Soc. 125 (2003) 10420-10428.

[13] R. Sarkar, P. Ahuja, P.R. Vasos, G. Bodenhausen, Long-lived coherences for homogeneous line narrowing in spectroscopy, Phys. Rev. Lett. 104 (2010) 053001.

[14] G. Pileio, M. Carravetta, M.H. Levitt, Extremely low-frequency spectroscopy in low-field nuclear magnetic resonance, Phys. Rev. Lett. 103 (2009) 083002.

[15] I. Bertini, M. Fragai, C. Luchinat, G. Parigi, H-1 NMRD profiles of diamagnetic proteins: a model-free analysis, Magn. Reson. Chem. 38 (2000) 543-550.

[16] A.S. Bornet, R. Sarkar, G. Bodenhausen, Life-times of long-lived coherences under different motional regimes, J. Magn. Reson. 206 (2010) 154-156.

[17] R.R. Ernst, G. Bodenhausen, A. Wokaun, Principles of Nuclear Magnetic Resonance in One and Two Dimensions, Oxford University Press, Oxford, 1987.

[18] J. Cavanagh, W.J. Fairbrother, A.G. Palmer, M. Rance, N.J. Skelton, Protein NMR Spectroscopy: Principles and Practice, Elsevier Science, 2007.

[19] M.H. Levitt, L. Dibari, Steady-state in magnetic-resonance pulse experiments, Phys. Rev. Lett. 69 (1992) 3124-3127.

[20] P. Ahuja, R. Sarkar, P.R. Vasos, G. Bodenhausen, Molecular properties determined from the relaxation of long-lived spin states, J. Chem. Phys. 127 (2007) 134112.

[21] R. Sarkar, D. Moskau, F. Ferrage, P.R. Vasos, G. Bodenhausen, Single or triple gradients?, J Magn. Reson. 193 (2008) 110-118.

[22] R. Sarkar, P. Ahuja, D. Moskau, P.R. Vasos, G. Bodenhausen, Extending the scope of singlet-state spectroscopy, ChemPhysChem 8 (2007) 2652-2656. 